Images dans le monde ibérique et ibéricoaméricain

5 | 2012

Le visible et l'invisible dans le monde hispanique et hispano-américain

\title{
Visibilité à l'esquive et invisibilité en latence pour un soldat de La Reddition de Breda
}

\section{Monique Plâa}

\section{(2) OpenEdition \\ Journals}

Édition électronique

URL : http://journals.openedition.org/agedor/868

DOI : 10.4000 /agedor.868

ISSN : 2104-3353

Éditeur

Laboratoire LISAA

Référence électronique

Monique Plâa, "Visibilité à l'esquive et invisibilité en latence pour un soldat de La Reddition de Breda », L'Âge d'or [En ligne], 5 | 2012, mis en ligne le 01 mars 2012, consulté le 16 décembre 2019. URL : http:// journals.openedition.org/agedor/868; DOI : 10.4000/agedor.868 
Monique PL $\hat{A} A$

Université Paris Est Marne-la-Vallée, LISAA (EA 4120), EMHIS

\title{
VISIBILITÉ À L'ESQUIVE ET INVISIBILITÉ EN LATENCE POUR UN SOLDAT DE LA REDDITION DE BREDA
}

\begin{abstract}
Résumé : Vélasquez a peint La reddition de Breda pour le Salon des Royaumes. Le tableau est unanimement considéré comme un des chefs-d'œuvre du Prado. Bien que la composition, la lumière, l'espace mais aussi la singularité de son doigt pointé vers le ciel lui donnent une importante toute particulière, le personnage au pourpoint blanc ne semble pourtant pas avoir particulièrement retenu l'attention de la critique. Dans la présentation qui suit, en prenant pour point de départ l'article que Angulo Íñiguez écrit en 1947, nous tenterons d'approcher la possible signification de ce personnage à la fois remarquable et mystérieux. Étant donné que Angulo Íñiguez met en évidence sans s'y arrêter particulièrement du reste - le caractère religieux des personnages dont Velázquez est parti pour élaborer le jeune homme au pourpoint blanc, nous nous demanderons si son index renvoie à une invisibilité spirituelle ou une invisibilité d'un genre peut-être moins transcendant mais, qui sait, plus intimement et secrètement relié à notre destin sur terre.
\end{abstract}

Mots-clés : Vélasquez - Breda - personnage - interprétation

Resumen: Velázquez pintó La rendición de Breda para el Salón de Reinos. El cuadro se ha convertido en uno de los más visitados y comentados del Museo del Prado. El personaje vestido del jubón blanco, a pesar de la importancia que le dan la composición, la luz, el espacio y también la singularidad de su dedo levantado hacia el cielo parece no haber llamado la atención de la crítica. En este trabajo, partiendo del artículo escrito por Angulo Íñiguez en 1947, intentaremos acercarnos al posible significado de esa figura llamativa y misteriosa. Dado que Angulo Íñiguez pone de manifiesto - aunque sin prestarle mayor atención - el carácter religioso de las figuras de las cuales partió Velázquez para elaborar al joven del jubón blanco, nos preguntaremos si su dedo índice remite a une invisibilidad espiritual o una invisibilidad quizás no tan convencionalmente trascendente, pero sí entrañable y ocultamente relacionada con el destino humano.

Palabras clave: Velázquez - Breda - personaje - interpretación 


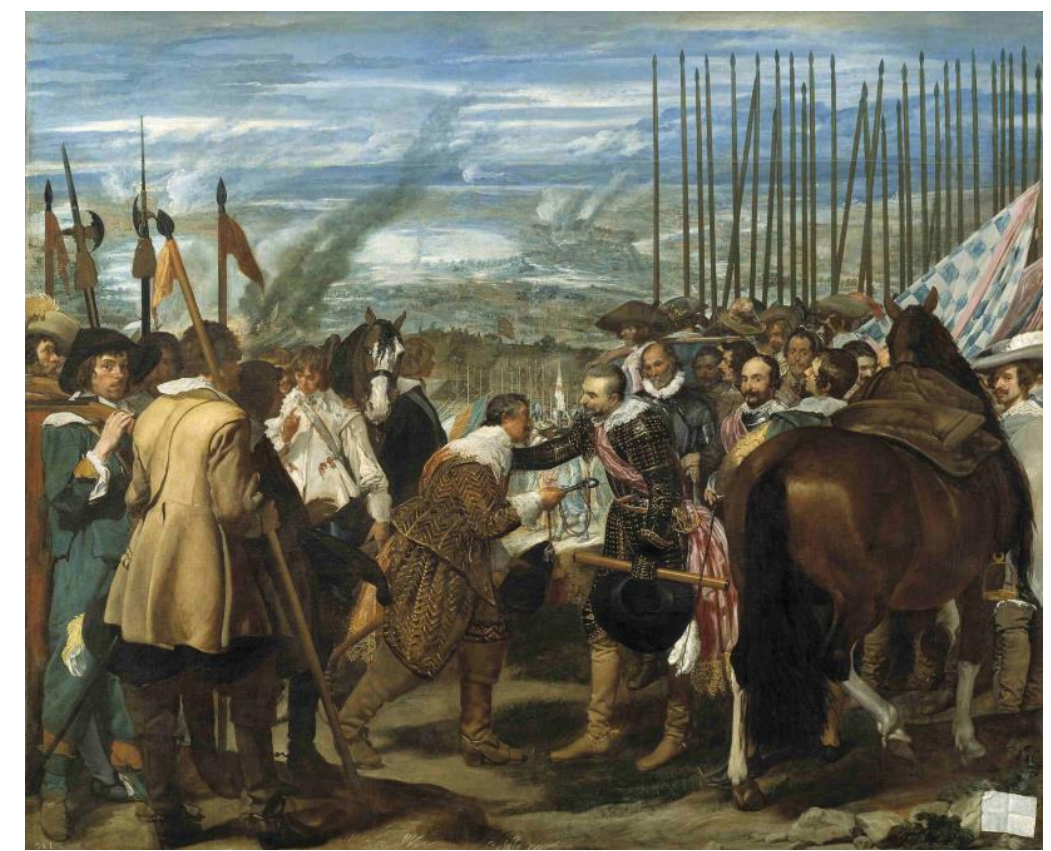

Diego Velázquez, La rendición de Breda, 1635, huile sur toile, 307 x 367 cm. Prado, Madrid

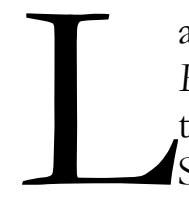

a lumière qui en peinture sous-tend la part de visibilité du tableau a, dans $L a$ Reddition de Breda, d'étonnantes caractéristiques. Elle vient de la gauche, comme en témoignent la casaque du Hollandais de l'avant-plan, la partie haute du visage de Spinola et aussi une partie de la tête ou des vêtements de quelques soldats. Mais, tandis que certains personnages sont partiellement éclairés, d'autres se trouvent plongés dans l'ombre : loin de toute objectivité naturaliste, la circulation de l'ombre et de la lumière s'avère surtout arbitraire. Par exemple, au tout premier plan, le travail sur les ombres au niveau du sol relève d'un effet "micro nuages »: la luminosité apparaît à un point quelconque puis disparait juste à côté pour réapparaitre à peine un peu plus loin. Sans doute, comme l'ont remarqué plusieurs critiques, le tableau y gagne-t-il en profondeur au sens propre mais vraisemblablement aussi, pourrait-on ajouter, au sens figuré du terme. La circulation arbitraire de la lumière sollicite le regard du spectateur qui alors passe d'une écharpe vibrante à une plume éclatante, d'une plume à une collerette, d'une collerette à une casaque et, quand ralentit la sollicitation qu'occasionne la succession des surgissements lumineux, le regard va plus essentiellement d'une casaque jaune à une lagune blanche, d'une lagune blanche à un pourpoint blanc; ce pourpoint que porte le jeune Hollandais placé quelque peu en retrait par rapport à Justin de Nassau mais face au spectateur. La blancheur du pourpoint, rehaussée par l'éclat du col de Justin de Nassau et surtout par l'éclat de la tête du cheval, offre, quoique sans ostentation - mais l'ostentation est toujours étrangère à l'art de Vélasquez -, une visibilité singulière à ce jeune Hollandais en concentrant la lumière sur lui plus que sur aucun autre personnage. Cette lumière attire d'autant plus le regard du spectateur qu'elle est sertie par des zones d'ombre qui, en ce point de la toile, intensifient, comme nulle part ailleurs dans le tableau, le contraste entre le clair et l'obscur; et le contraste se réalise ici au bénéfice du clair. Outre la lumière, la composition aussi mène le regard vers ce personnage. À l'exception de Justin de Nassau et d'Ambrosio Spinola, les héros au centre de la toile, et à l'exception aussi des deux Hollandais de l'avant-plan gauche, 
ce personnage est le plus visible parce que, contrairement à la plupart des hommes qui l'entourent, il est placé face au spectateur et parce que, après Justin de Nassau et Ambrosio de Spinola, c'est à lui qu'objectivement est réservé le plus d'espace. Figuré dans un centre évidé, serti par les ombres, point de recentrement de la lumière, le jeune homme au pourpoint blanc semble placé là pour solliciter et interroger le regard du spectateur. Dans l'œuvre de Vélasquez, il y a de nombreux visages dont le traitement est comme «suspendu ». Dans le prolongement de la dernière époque du Titien, Vélasquez se libère, dès le début de sa carrière, de la contrainte du fini en jouant sur les possibilités de l'inachevé comme c'est le cas ici : l'ébauche du visage du jeune homme est précise mais la finition est imprécise. Le visage est éclairé par une lumière dense et singulièrement mouvante de sorte que la tension entre le fini esquivé et la vibration lumineuse donne une présence forte à ce personnage que Vélasquez semble tout à la fois montrer et ne pas montrer, signaler et occulter. Dans cet entre-deux d'une visibilité qui s'affiche et s'oblitère, le jeune homme au pourpoint blanc parait étrangement mystérieux.

La critique, quand elle prend en compte ce personnage, l'identifie comme soldat ${ }^{1}$. Mais d'abord, est-on bien sûr qu'il s'agisse d'un soldat ? Son habit est tout à fait différent de celui des miliciens de base du premier plan. Il ne semble pas non plus faire partie des officiers : il ne porte pas d'écharpe de commandement, d'ailleurs, il a l'air bien jeune pour commander, en plus, dans son porte-épée il n'y a aucune épée visible; quant à son pourpoint, par la couleur blanche, extrêmement rare sur les champs de bataille, par la somptuosité de la coupe, des matières et des broderies, il fait davantage penser à un habit d'apparat et il contribue à rendre le jeune homme encore plus singulier et mystérieux puisque, dans le camp des Hollandais, en dehors de Nassau, on ne voit que de simples soldats. Mais le comble de l'étrangeté dont semble nimbé le jeune homme au pourpoint blanc vient de son geste, de sa main verticale, de ce doigt levé que Velázquez rend flagrant par la présence d'une ombre exceptionnellement précise qui dédouble la main sur le pourpoint.

La peinture italienne, celle du Tintoret et de Véronèse en particulier, a produit de multiples tableaux qui réunissent des scènes diverses plus ou moins librement enchâssées dans la composition d'ensemble. Velázquez a fait, dès Los borrachos, l'expérience d'une polyfocalité narrative où les séquences périphériques s'insèrent sans contrainte forte dans la scène principale. Dans La reddition de Breda, tandis qu'au centre visuel et sémantique du tableau, Justin de Nassau, le gouverneur de Breda, remet les clefs de la ville à Ambrosio de Spinola, le chef génois des armées espagnoles, le regard des soldats se tourne fréquemment vers autre chose, vers le spectateur, vers des compagnons d'armes, vers le fond et vers on ne sait exactement quoi. Mais la présence du jeune homme au pourpoint blanc ne se laisse pas réduire à un de ces effets de décentrement qui abondent dans la peinture de Vélasquez, et ce, d'autant moins que l'homme au doigt pointé semble retenir l'attention d'un de ses compagnons et, fait plus susceptible encore d'intriguer le spectateur, il parait aussi retenir l'attention du cheval qui est à sa droite et dont Vélasquez a traité la tête avec un soin extrême, comme s'il s'agissait d'un portrait. De sorte que l'ensemble constitue une scène spécifique et repérable qu'il est malaisé de raccorder à la scène principale; en effet, quel rapport peut-il bien y avoir entre la scène centrale de la remise des clés et le jeune homme au pourpoint blanc et à l'index pointé vers le haut ? C'est à cette question que nous allons

\footnotetext{
${ }^{1}$ Cf. BROWN, Jonathan, Velázquez. La técnica del genio; GUDIOL, José, Velázquez 1599-1660 : sa vie, son œuvre, son évolution, Paris, Éditions Cercle d'art, 1975 ; LÓPEZ-REY, José, Velázquez : catalogue raisonné, Köln, Taschen, 1999.
} 
tenter de répondre et pour cela nous nous demanderons d'abord qui peut bien être ce jeune homme au pourpoint blanc.

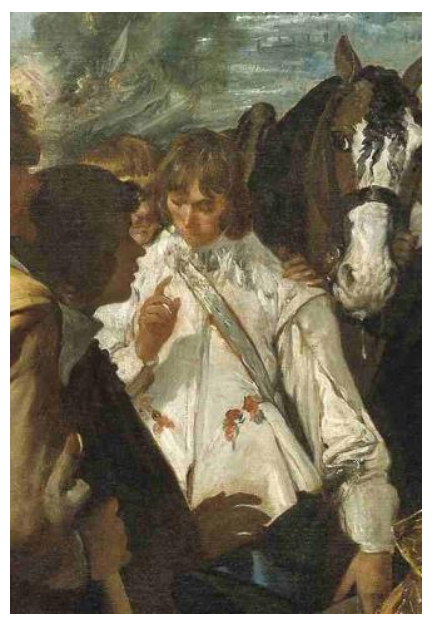

Diego Velázquez, La rendición de Breda, 1635, huile sur toile, 307 x 367 cm, détail. Prado, Madrid

Diego Angulo Íñiguez, critique d'art espagnol dont les publications paraissent en Espagne à partir des années 40 , a réalisé sur ce tableau un travail particulièrement attentif. Angulo Íñiguez ne s'intéressait pas à priori au jeune homme au pourpoint blanc mais il l'a, pour ainsi dire, croisé en suivant la logique de son approche de la peinture. Cette logique se fonde sur une idée lumineusement simple : un peintre réalise un tableau en convoquant les tableaux qu'il a déjà vus, ce dont, en principe, personne ne saurait douter. Aussi, dans l'article qu'il consacre à Vélasquez, en 1947, la proximité que Angulo Íñiguez crée, par son écriture, entre lui et Vélasquez n'a pas pour but de chercher l'homme dans le peintre, mais d'approcher au plus près l'artiste pour retrouver la piste des tableaux dont son œil s'est nourri avant que sa main ne les incorpore dans l'œuvre en cours :

Quien lea las páginas que siguen le verá hojeando el «Libro de dibujos y estampas grande » y el «Arte de la escultura » del Buonarrota que figuran en el inventario de su librería, en busca de reproducciones donde inspirarse : podrá contemplarle, además, recorriendo las galerías reales con el mismo fin : y hasta presenciará cómo transforma esos modelos haciéndoles sufrir maravillosas metamorfosis dignas de los dioses del Olimpo, para terminar convirtiéndolos en hermosísimos lienzos que se llaman la Rendición de Breda o las Hilanderas².

Au départ des «merveilleuses métamorphoses » qui allaient mener à La reddition de Breda, il y aurait une gravure de médiocre facture du français Bernard Salomon. De cette gravure, intitulée Abraham et Melquisedec, Vélasquez aurait emprunté la distribution des personnages en deux groupes faussement symétriques, puisque celui de droite est de fait, dans La reddition de Breda, beaucoup plus fourni que celui de gauche ; il aurait aussi adopté l'idée de fermer le premier plan par la verticalité des lances sur le côté droit ; enfin, Vélasquez aurait également retenu de la gravure de Salomon la mise en évidence de l'échange central par l'évidement du premier plan qui laisse voir, au deuxième plan, le défilé

\footnotetext{
2 ANGULO ÍÑIGUEZ, Diego, Estudios completos sobre Velárquez, Madrid, Centro de Europa Hispánica, 2007, p. 65.

Dans ce recueil d'articles, celui qui sera abondamment repris ici c'est : «Velázquez: cómo compuso su principales cuadros », publié en 1947 à Séville.
} 
des militaires ${ }^{3}$. Mais, selon Angulo Íñiguez, c'est dans l'Expolio du Greco et dans Le Christ et le centurion de Véronèse que Vélasquez a trouvé la base à partir de laquelle il allait élaborer de nombreux personnages de La reddition de Breda. Alors que l'Expolio aurait alimenté la réalisation de la partie droite de La reddition de Breda, Le Christ et le centurion de Véronèse serait la base invisible de diverses figures de la partie gauche et, en particulier, du jeune homme au pourpoint blanc.

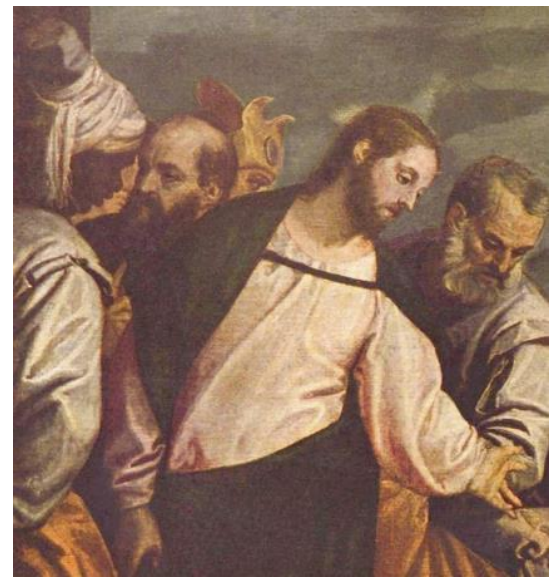

Paul Véronèse, Le Christ et le centurion, 1571, huile sur toile, 192 x 297 cm, détail. Prado, Madrid

D’abord, Angulo Íñiguez porte son attention sur le personnage de race noire qui figure à l'extrême gauche du tableau de Véronèse. Vélasquez, à partir de ce personnage aurait créé deux soldats, les deux traités dans l'ombre et même, pour le deuxième quasiment comme une ombre, l'un sera placé à l'avant, l'autre immédiatement derrière le jeune homme au pourpoint blanc. Vélasquez aurait également tiré profit de la puissante épaule du noir enturbanné dont il aurait repris et accentué le contour saillant pour dessiner le Hollandais de dos à l'avant-plan de La reddition de Breda. Ensuite, le jeune homme au pourpoint blanc, précisément, résulterait non plus d'un personnage dédoublé - comme dans l'opération précédente - mais de deux personnages fusionnés : il viendrait de la fusion de l'apôtre au doigt pointé vers le haut et du Christ au vêtement lumineux dont le corps occupe, dans le tableau de Véronèse, une position frontale par rapport au spectateur.

Si el negro del pintor veneciano lo desdobla en dos personajes, en el soldado del jubón blanco funde, en cambio, las figuras del Salvador y del apóstol con la mano en alto del Veronés. Debe al primero, sobre todo, la luminosidad de su vestidura, en cierto grado la frontalidad del cuerpo, y el brazo, que ligeramente inclinado sostiene la bolsa dirigiéndose hacia el centro del cuadro. Del apóstol procede esa mano con el índice en alto, que con el perfil en sombra de su compañero tanto contribuye a producir la sensación de espacio dentro del grupo ${ }^{4}$.

Sur la lancée des métamorphoses invisibles, Angulo Íñiguez observe que du bras droit du Christ qui rencontre le bras de l'apôtre Pierre dans Le Christ et le centurion est sans doute née la tête du cheval dont le blanc éclatant est nécessaire pour la composition du groupe de gauche de La Réddition de Breda. Devant ces métamorphoses en cascade, Angulo

\footnotetext{
${ }^{3}$ Cette composition, à quelques détails près, est aussi retenue par l'élève de Vélasquez, Jusepe Leonardo pour la réalisation du tableau Socorro de Brisach qui devait illustrer également le Salon des Royaumes.

${ }^{4}$ ANGULO ÍÑIGUEZ, Diego, Estudios completos..., op. cit., p. 86.
} 
Íñiguez lui-même ne manque pas de s'émerveiller : les métamorphoses de Vélasquez, écritil, sont merveilleuses puisque, comme au plus beau temps de la mythologie, on y voit des humains transformés en animaux - «Como en los mejores tiempos de la mitología griega vemos a dos criaturas humanas convertirse en dos poderosos corceles $»^{5}$.

Le travail d'analyse de Angulo Íñiguez impose deux commentaires. D'abord, bien entendu, l'enthousiasme ne vaut pas pour démonstration mais il faut reconnaitre l'originalité d'une démarche qui, d'ailleurs, révèle davantage une sensibilité d'artiste que de critique; ensuite, dans la logique de ce constat, on remarque que l'attention portée par Angulo Iñiguez à l'élaboration, à la métamorphose des formes, ne fait aucun cas des enjeux de sens dont ces formes pourraient être le support : le doigt qui vient d'un apôtre en contrepoint de la tête sombre qui vient d'un Noir, n'ont d'autre intérêt, dans l'approche proposée par Angulo Íñiguez, que de créer, au terme de la métamorphose réalisée par Vélasquez, une "sensation d'espace dans le groupe ». Que le jeune homme au pourpoint blanc soit le résultat de la fusion de deux personnages qui figuraient un apôtre et le Christ, pour Angulo Íñiguez, qui capte avant tout les formes et les lumières, n'a pas plus d'intérêt que s'il était né d'un cheval et d'un parfait inconnu. Et, pourtant, pourtant, une si noble ascendance, si vénérablement connotée, un apôtre et le Christ, par-delà la métamorphose des formes, ne pourrait-elle faire quelque peu sens pour un personnage si mystérieusement visible?

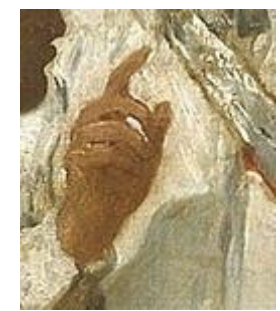

Diego Velázquez, La rendición de Breda, 1635, huile sur toile, 307 x 367 cm, détail. Prado, Madrid

Dans l'espoir, quoique frêle, de trouver un étayage de sens pour le jeune homme au pourpoint blanc, on va maintenant recentrer l'analyse sur ce qui semble être un détail significatif : l'index pointé. En 1997, Daniel Arasse s'intéresse au sujet dans le tableau et il consacre un article à l'extraordinaire index du Moïse de Michel-Ange, index qui, comme on le sait, se perd dans la barbe qu'il retient ${ }^{6}$. Arasse atteste de la codification du geste de l'Antiquité à nos jours: geste d'autorité, geste de bénédiction, geste d'injonction, geste d'annonciation, de méditation ou de mélancolie, etc. : il suffit d'un rien pour que le sens de l'index pointé change du tout au tout. Dans une démarche menée en confrontant les multiples traces iconographiques mais aussi textuelles qui entourent et précédent la réalisation de l'index de Moïse, Daniel Arasse arrive à la conclusion que, dans cet index qui file dans la barbe, il y a une forme de l'autoportrait : une manière immensément ambiguë de représenter le pouvoir. Comme Moïse, qui n'atteindra jamais la terre promise, le créateur Miche-Ange, nous explique Arasse, aurait lui aussi un sentiment de frustration et, pareil à Moïse soumis aux lois de Dieu, Michel-Ange se sentirait également contraint de se soumettre au pouvoir de la conscience et au pouvoir du Pouvoir, qui se trouve être, en dernière instance, celui du donneur d'ordre qui finance le peintre. À défaut d'être en

\footnotetext{
${ }^{5}$ Ibid.

6 ARASSE, Daniel, "Michel-Ange et l'index de Moïse », Le Sujet dans le tableau. Essais d'iconographie analytique, Paris, Flammarion, 1997, p. 72-90.
} 
mesure de mener à bien ce genre de démarche avec le doigt du jeune homme au pourpoint blanc, on se contentera ici d'établir deux constats.

D'abord, il faut prendre acte du fait que le geste qui consiste à pointer un doigt est fréquent dans la peinture du temps de Vélasquez mais, dans la très grande majorité des cas, le doigt fonctionne comme un simple surlignage: l'index d'un personnage pointe le personnage le plus important du tableau ou il pointe ce qui, bien que figuré au deuxième plan, organise pourtant le sens de la scène du premier plan - c'est justement le cas dans certains tableaux du programme de peintures du Salon des Royaumes dont La reddition de Breda fait partie, ou bien l'index sert à montrer un emblème comme il arrive dans de nombreux tableaux où saint Jean Baptiste pointe du doigt un agneau miniature posé sur son livre. Le deuxième constat concerne, par contraste, la rareté d'un index qui, orienté verticalement, vers le haut, pointerait un au-delà du tableau. D'ailleurs, ces seules indications de hauteur et d'au-delà, sollicitent immanquablement et d'entrée notre imagination religieuse. Et, en effet, ce geste semble réservé à des cas bien particuliers : à saint Jean-Baptiste, à l'Ange de l'Annonciation ou, parfois, à un apôtre - comme dans le tableau de Véronèse -, ou au Christ quand, par exemple, le Christ rappelle au traitre la présence du Père. Ce n'est que très exceptionnellement, et qui sait, d'ailleurs, s'il ne s'agit pas d'un cas unique, qu'on trouve l'exemple d'une peinture dont le protagoniste à l'index pointé vers le haut n'est ni le Baptiste, ni l'Ange de l'Annonciation, ni le Christ, ni même un personnage des Evangiles mais un militaire. Peint pour Philippe II, le sujet de ce tableau du Titien dont le héros est un militaire est contenu dans le titre: Allocution d'Alfonso d'Avalos à ses troupes. D'Avalos, le chef des armées de Charles V en Italie, avait la réputation d'être un flamboyant soldat au service de Dieu. Et, en effet, la composition du tableau, la sérénité de l'ensemble, le page que l'on pourrait confondre avec un ange, la complémentarité visuelle et sémantique de la pique, du bâton de commandement et du doigt pointé vers le haut, vers un au-delà du tableau, font un ensemble iconographique dont le sens s'impose : les armes se doivent d'être et sont au service de Dieu. De sorte que le geste du militaire du Titien ne serait pas si éloigné de celui de l'apôtre ou du Christ qui renvoient leur interlocuteur, et avec lui le spectateur, à la présence invisible mais transcendante du Dieu suprême, et le geste du militaire s'avérerait aussi très proche de celui de l'Ange de l'Annonciation et de saint Jean-Baptiste qui tous deux anticipent la venue du Christ parmi les hommes.

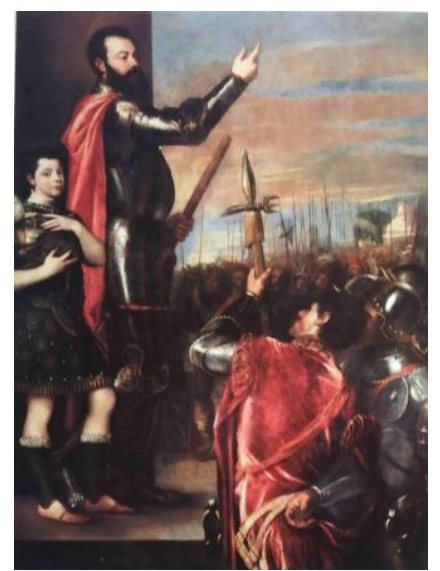

Titien, Allocution d'Alphonse d'Avalos à ses soldats, 1540-41, huile sur toile, 223 x 165 cm. Prado, Madrid

Ainsi donc, il semblerait que, même pointé par un personnage qui exceptionnellement n'est pas religieux, le doigt vers le haut, a de toute façon un sens religieux. Vélasquez a procédé à l'inverse du Titien. D'Avalos, quoique militaire et non 
religieux, est représenté d'une manière qui s'inscrit si fermement dans la tradition des personnages délivrant avec leur doigt pointé vers le ciel un message religieux que, pour le spectateur, l'interprétation ne peut faire aucun doute. Avec le jeune homme au pourpoint blanc, tellement présent mais peint comme en retrait, représenté parmi les militaires mais ne leur ressemblant en aucun point, Vélasquez décline en mode mineur le motif du doigt pointé vers le ciel : la tête inclinée, le bras replié et le doigt légèrement incurvé du jeune homme n'offrent à celui qui regarde le tableau qu'une visibilité à l'esquive. Mais puisque, d'une part, le doigt pointé vers le haut semble constamment codifié dans un sens qui renvoie à l'imminence du divin ou à l'invisibilité de la transcendance religieuse et puisque, par ailleurs, dans ses spéculations, Angulo Íñiguez affirme que les ascendants artistiques du jeune homme au pourpoint blanc sont un apôtre et le Christ, ne pourrait-on supposer que le personnage est porteur d'une invisibilité en latence? D'une invisibilité de nature religieuse. Pour consolider cette supposition, il faudrait que Vélasquez ait traité le thème de l'index pointé à d'autres reprises et sous diverses formes, ce qui n'est pas exactement le cas.

Dans l'œuvre de Vélasquez, il n'y a apparemment, sous réserve de recherches plus approfondies, que quatre tableaux qui puissent, peu ou prou et par comparaison, éclairer le geste du jeune homme au pourpoint blanc : Le Christ chez. Marthe et Marie, Saint Jean-Baptiste du Désert. Le Christ à la colonne et La forge de Vulcain. Dans le premier tableau, peint en 1618, c'est une femme du peuple qui au premier plan oriente par son index pointé horizontalement l'attention d'une jeune femme en train de cuisiner, et par contre coup l'attention du spectateur, vers le deuxième plan où se trouvent le Christ, Marthe et Marie. Le geste est évident mais son interprétation est en partie tributaire de l'identité que l'on prête aux deux femmes du premier plan : si les deux femmes du premier plan, comme on tend généralement à le croire, dédoublent Marthe et Marie qui accompagnent le Christ au deuxième plan, alors le doigt pointé inscrit fortement au niveau de la vie quotidienne la nécessité de soumettre la vie matérielle à la vie spirituelle. Le deuxième tableau, Saint JeanBaptiste du Désert, est simplement conventionnel et, d'ailleurs, il n'est généralement plus attribué à Vélasquez. Le Christ à la colonne montre un ange dont la main ressemble à celle du jeune homme au pourpoint blanc, seule diffère l'orientation du doigt qui, dans Le Christ à la colonne, est pointé vers le bas pour signaler le Christ assis à même le sol. Dans La forge de $V$ ulcain, Apollon, lumineux et nimbé, et semblant de son doigt orienté vers le ciel prendre les dieux à témoin, invite à la prudence : certes, Apollon est un messager et son message est bien une annonce divine mais la teneur de son annonce n'en est pas moins triviale. Ainsi donc, il apparait que Vélasquez a représenté peu de fois ce geste et que chaque fois qu'il l'a représenté, la part divine est traitée différemment: avec gravité dans le quotidien de la cuisine, avec affliction quand le fils de Dieu fait homme est à terre, avec plus de distance quand le messager des dieux transmet son message divin et prosaïque. Tant de nuances invitent à ne se risquer qu'avec une extrême prudence dans une interprétation religieuse du geste du jeune homme au pourpoint blanc. En revanche, un autre tableau, La tentation de saint Thomas d'Aquin, peint en 1632, pourrait nous encourager à en prendre le risque. Dans La tentation de saint Thomas d'Aquin, il n'y a pas de doigt pointé vers le ciel mais l'ange qui soutient le saint, par son profil allongé, par le tracé très droit de son nez et de sa bouche, par l'imprécision du traitement du visage et la clarté de la carnation, n'est pas sans rappeler le jeune homme au pourpoint blanc, jeune homme auquel sa quasi-blondeur, une certaine douceur, l'illumination du visage et, dans une certaine mesure, la blancheur du vêtement, pourrait, en effet, donner aux yeux du spectateur décidé à aller dans ce sens, l'air d'un ange. Si l'on veut se risquer à supposer que le jeune homme au pourpoint est, tel un ange, le messager de quelque annonce, la question qu'il faudrait alors poser avec prudence serait : de quelle annonce peut-il bien être le porteur? 
On ignore tout des consignes qui ont été données aux peintres dont les douze tableaux -l'un des douze allait devenir La reddition de Breda - devaient décorer le Salon des Royaumes. La pièce écrite par Calderón en 1625, El sitio de Breda, peut toutefois fournir quelques indications sur la manière dont l'événement était interprété quelques mois seulement après qu'il ait eu lieu. El sitio de Breda est un hymne à la royauté espagnole qui met son armée au service de la cause chrétienne. Dans cette pièce, l'adversaire c'est l'hérétique tandis que les soldats de Philippe IV sont les défenseurs de la religion catholique. De cette œuvre quelque peu rudimentaire, Vélasquez a retenu le portrait de Spinola en homme respectueux de l'adversaire puisque, déclare dans la pièce le chef de l'Armée de Philippe IV, la valeur du vaincu fait la gloire du vainqueur. En revanche, on ne saurait trouver dans La reddition de Breda absolument rien qui relèverait de la stigmatisation de la religion de l'autre. Du reste, alors que dans la pièce de Calderón, la victoire du Roi est fortement liée à la victoire de la Foi, aucun des peintres qui ont travaillé pour le Salon des Royaumes ne semble s'être soucié de figurer le triomphe de la Foi: les peintres ont privilégié la célébration du chef des armées et l'action épique menée au service de Philippe IV et pour ses Royaumes, précisément. Mais il y a une unique et tout à fait extraordinaire exception. Trois peintres du roi, Crescenzi, Maíno et Vélasquez, avaient la responsabilité d'engager ceux qui, parmi leurs collègues, auraient à peindre les douze toiles qui allaient orner les murs du Salon. Maíno et Vélasquez ont également peint chacun un tableau. Or, le tableau de Maíno est pour le moins spectaculaire : il relègue délibérément la célébration du roi, du favori du roi, et du chef des armées du favori du roi au deuxième plan tandis que le premier plan est tout entier occupé par une scène de charité chrétienne qui, par le traitement donné au motif principal, n’est pas sans rappeler la tradition des piétés. Mais Maíno ne se contente pas de placer une scène religieuse de manière prioritaire, il contrevient délibérément à ce qu'on croirait être les attendus d'un tableau destiné à célébrer le Royaume. En effet, le type des personnages du premier plan, leur blancheur notoire et surtout le bleu et l'or de leurs vêtements font penser que le blessé et tous ceux qui l'entourent ne sont pas les vainqueurs, les catholiques, mais bien plus probablement, les vaincus, les protestants. Si Maíno, moine franciscain, a eu l'audace de voir dans les vaincus des hommes de Dieu sur la terre - et ce faisant d'aller à l'encontre de la vision manichéenne telle que la suggère la pièce de Calderón mais aussi telle que la donnent à voir l'ensemble des peintres qui ont contribué à la décoration du Salon des Royaumes -, ne peut-on se risquer à penser que Velázquez, de manière plus sibylline, a fait de même : le jeune homme au pourpoint ne porterait-il pas un invisible message non plus tourné vers le ciel, mais bien davantage vers la terre, moins destiné aux vainqueurs qu'aux vaincus?

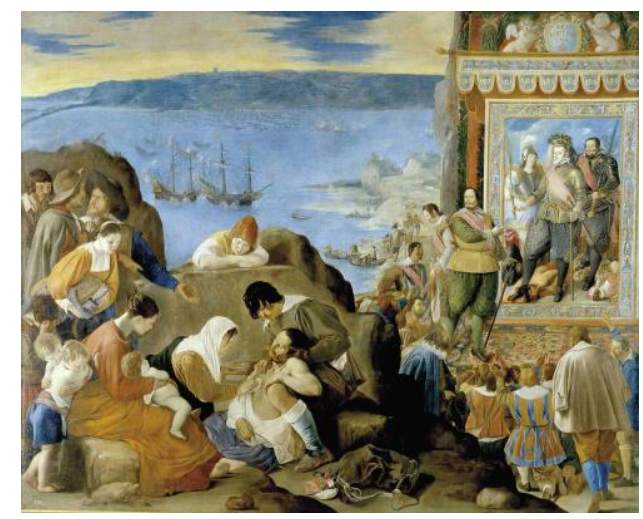

Jean-Baptiste Maíno, Reprise de Babia, 1634-1635, huile sur toile, 309 cm x $381 \mathrm{~cm}$. Prado, Madrid. 
Dans le texte singulièrement subtil qu'il consacre à Vélasquez, Claude Esteban, remarque que, dès ses premières œuvres, Vélasquez pare le quotidien le plus humble d'une discrète sacralité qui fait d'un mendiant un apôtre et de l'acte de boire, une scène liturgique. Lorsqu'il considère $L a$ reddition de Breda, Claude Esteban signale, comme le fait unanimement la critique, l'élégance du geste du vainqueur, «comme s'il oubliait sa victoire et ne désirait reconnaître chez son adversaire que la seule vaillance » mais il remarque aussi les lances « qui barrent l'horizon comme une menace toujours vive » et il célèbre également, la place, en effet extraordinaire, du cheval du premier plan, « longue plage brune et paisible, qui, parle de rémission, peut-être de concorde ${ }^{7}$. Le jeune homme au pourpoint blanc est dans le camp des vaincus, entouré par les vaincus. Breda avait été reconquise en 1625, année de gloire des armées des Royaumes, mais en 1635, alors qu'Olivares donne l'ordre de réaliser le vaste programme de décoration du Salon emblématiquement nommé des Royaumes, la domination de l'armée espagnole est déjà fragilisée sur plusieurs fronts et Breda ne tardera pas à repasser dans le camp des protestants et les vainqueurs de 1625 seront les vaincus de 1635 . Peut-être pourrait-on alors se risquer à penser que la visibilité de l'étrange jeune homme au pourpoint blanc parle, pour le dire avec les mots de Claude Esteban, moins d'un dieu invisible dont on annoncerait avec certitude la venue triomphale, que d'un destin humain tout aussi invisible mais frappé irrémédiablement d'incertitude. On pourrait alors croire que, pareil à la longue plage brune et paisible du cheval du premier plan remarquée par Claude Esteban, le jeune homme au pourpoint blanc parle aussi de rémission et de concorde, et qui sait, s'il ne parle pas d'abord et surtout, à l'heure où le bleu du ciel se mâtine des gris fuligineux du dernier incendie, d'une invisible mais si nécessaire consolation quand le destin des hommes est pris dans les aléas du temps.

Quoique pris dans le tranquille équilibre qui, dans le monde de Vélasquez, harmonise toute chose, le jeune homme au pourpoint blanc est singulièrement visible par la lumière qui l'éclaire et par l'espace qu'il occupe ; sa visibilité est rehaussée par l'index levé, dont la nature codifiée est d'être un signe visible qui, lorsqu'il pointe vers le haut un au-delà du tableau, sollicite notre imagination de la transcendance invisible ce qui, par un hasard qui pourrait bien ne pas en être un, ferait résonance avec l'ascendance artistique que prête Angulo Íniguez au jeune homme au pourpoint.

Mais le code, tel qu'on peut le déduire de l'ensemble des peintures du Salon des Royaumes, veut que l'on exalte la gloire des Armes et qu'on taise la Foi qui est pourtant la cause et la justification des batailles. Peintre du roi, comme Velázquez, et comme lui responsable du choix des peintres dont les œuvres décoreront le salon des Royaumes, Maíno contrevient manifestement aux attendus du tableau de bataille : c'est la religion qu'il met au premier plan, non pas celle qu'impose un Dieu tout-puissant et invisible mais la charité chrétienne incarnée par les hommes et pour les hommes.

En toutes choses, Velázquez est le peintre des nuances infiniment subtiles, de l'esquive suggestive. Le jeune homme au pourpoint blanc semble visible pour mieux jouer avec l'invisibilité et, à l'heure où la gloire s'éloigne des Armes et des Royaumes, sa blancheur nimbée de lumière et de mystère pourrait être le signe d'une discrète compassion offerte aux vaincus et, qui sait, une parole de consolation offerte, par-delà les agitations de ce monde, à chacun des spectateurs de La reddition de Breda.

\footnotetext{
${ }^{7}$ ESTEBAN, Claude, Trois Espagnols. Velázquez, Goya, Picasso, Paris, Farrago, 2000, p. 23.
} 